\title{
Examination of Specific Gravity of Urine Related to Chronic Kidney Diseases Based on MEMS and Data Mining
}

\author{
Dr. Narendra B. Mustare \\ ${ }^{1}$ Professor, CVR College of Engineering/EIE Department, Hyderabad, India. \\ Email: narendra@cvr.ac.in
}

\begin{abstract}
Chronic Kidney Disease (CKD) is a major public health problem with growing challenges for its early diagnosis, timely prevention and effective treatment. The examination of specific gravity of urine in respect to CKD, like nephropathy and the parenchymal lesion is of great clinical importance for the treatment of the patient. The diagnosis of the doctor is based on accurate results in respect of the status of the specific gravity of urine. Mems is an effective and accurate tool for the measurement of the specific gravity of the liquid and thus the simulated results of the Mems are of importance in respect to the CKD mentioned above. The trend of the diseases can be correlated with the simulated output for which classification is done in the data mining software DTREG for helping the physicians so that they can prescribe the treatment of the subject in a precise manner.
\end{abstract}

Index Terms: CKD, Specific gravity of urine, MEMS and data mining.

\section{INTRODUCTION}

Nephropathy issues within the Udhanam region of Srikakulam district, Andhra Pradesh in India, have intended researchers to examine the assorted factors associated with kidney Diseases. Here, huge amount of farmers in agronomy are affected by Chronic Kidney Disease (CKD) [1]. It has been observed that the majority of people who are affected by this kidney disease in the Uddanam region are agricultural workers. In 2013, this CKD of the Uddanam region was named Uddanam nephropathy at the International Congress of Nephrology in China. The previous studies report states that there is a relationship between alteration of the gut microbiome (biological factor) and renal failure [2]. The Kidney is a very important regulator of equilibrium by filtration, organic process, secretion, synthesis, and degradation of metabolites in numerous pathways. Chronic means long-lasting, and CKD has been outlined based on the presence of kidney harm and its structure or faded kidney function or kidney damage and diminished function that lasts longer than three months. CKD is a serious public health issue with a universal prevalence of $13.4 \%$ and a mortality rate of 1.2 million (approx.) per year [3]. Chronic Kidney Disease (CKD) may be a worldwide health concern rising at a really quick pace. According to the National Kidney Foundation, one-tenth of the overall world population is affected by CKD leading to an increased death rate. It is estimated that in developing countries like China and India, the cases of kidney failure will increase disproportionately since the number of elderly people is increasing due to enhanced life span [4]. Chronic Kidney Disease is also termed end-stage renal disease.

So, for the diagnosis of CKD diseases like nephropathy and parenchymal lesion, it is necessary to examine and thereby measure the specific gravity of urine [5]. The prevailing approaches of measuring the specific gravity of urine are time-consuming and involve the chances of human error. Modern techniques are needed to stop the pathogens which are responsible for CKD. With the help of various engineering techniques, one can easily design controllers to assess as well as to prevent CKD permanently. A large amount of work has been done in medical sciences in the area of CKD, but there is still scope for further research. From the recent studies, advanced tools such as data mining, etc., are considered to be the current trend in the area of CKD.

In this paper, we have introduced a new method to measure the specific gravity of urine using MEMS. The principle of a cavity resonator is being used in our method [6]. The specific gravity of the urine (i.e., the concentration of solute in urine) is measured by the Micro-Electro Mechanical System (MEMS). For minimum specific gravity, the electrodes of MEMS will vibrate with maximum amplitude and thus the amplitude of the vibration of the electrode is functionally related to specific gravity which is the underlying principle of MEMS. In specific gravity methodology, the higher the specific gravity of the fluid, the lower the resonant frequency of the filled tube. The specific gravity sensor contains a chip level, vacuum prepacked, and resonant semiconducting material tube. Whereas the tube, filled with a fluid, is driven into resonance electrostatically and its motion detected capacitively. Data obtained from the sensor is fed to software for further examination of blood samples [7].

\section{RELATED WORK}

In the recent past, various methods, as well as their applications, have been developed to predict CKD. In what follows, we will review and investigate related works on classification methodologies for kidney diagnosis done previously.

In the field of medical diagnosis of chronic kidney disease, D. Noia et al. presented a classifier based on the ensemble of ten artificial neural networks with the data collected over a period of 38 years [8]. A software tool has been developed predicting the end-stage kidney disease 
(ESKD) risk of the patients as an online web application as well as an Android mobile application.

Gunasundari et al. proposed two modified Boolean Particle Swarm Optimization (BoPSO) algorithms viz. Velocity bounded BoPSO (VbBoPSO) and Improved Velocity bounded BoPSO (IVbBoPSO) for solving the problem of feature selection [9]. Both these algorithms have been tested on 28 benchmark datasets. The proposed system selects exclusive features from the datasets to achieve high classification accuracy.

In order to detect CKD, Salekin and Stankovic, in their paper, explained classification with feature selection methodology based on three classifiers namely K-nearest neighbor, random forest, and neural networks [10]. With the feature reduction methods namely, wrapper method and LASSO regularization, 12 attributes from 24 attributes have been selected to detect accuracy with high accuracy. Further, CKD detection has been done by reducing the number of attributes to 5 .

Using the WEKA data mining tool, Arora and Sharma focused on CKD detection with eight classification methods namely SGD, Random Subspace, SMO, JRIP rules, Hoeffding tree, Naive Bayes, Locally weighted learning [11]. The various performance trials of the resulting algorithms have also been presented.

Rubini and Eswaran propose three classifiers viz. radial basis function networks, multilayer perceptron, and logistic regression for the prediction of CKD [12]. Various performance metrics have also been calculated for the CKD dataset.

Gadaras and Mikhailov demonstrated a fuzzy classification method for the extraction of fuzzy rule sets from the dataset for the building of a medical diagnosis framework [13]. The proposed method is compared with the existing methods on three medical datasets namely Wisconsin breast cancer dataset, Pima Indian diabetes dataset, and the Bupa liver dataset.

Krause et al. showed an overview on the role of exosomes in kidney growth and diseases like renal cancer [14]. The review also includes recent research on the importance of exosomes as diagnostic markers and their therapeutic use in kidney diseases and cancers. The authors have also illustrated the proteins found in human urinary exosomes existing in the regions of the kidney and also about the significant genes found in exosomes of the various species.

Jella et al examined the regulation of epithelial sodium channels $(\mathrm{ENaC})$ in mpk CCD cells [15]. Glycolytic enzyme glyceraldehyde-3-phosphate dehydrogenase (GAPDH) was located within exosomes that were derived from proximal tubule LLC-PK1 cells. The study illustrates the importance of exosomes in the modulation of EnaC activity and in the

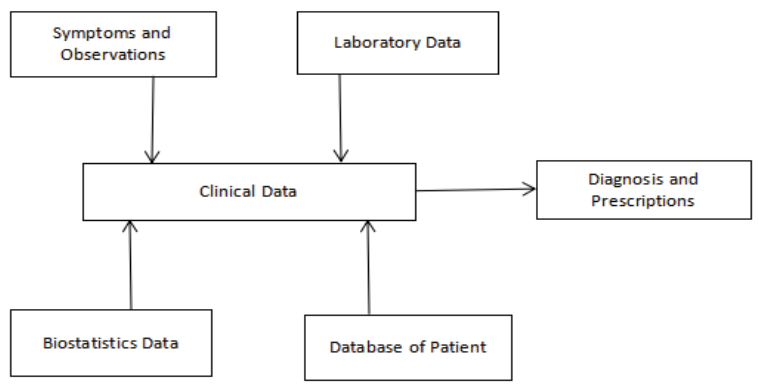

Figure 1. Existing System

\section{EXISTING SYSTEM}

The Existing system is shown in figure. 1 below.

It is a manual process which consumes 2-3 days to test the urine from micro array expression data [16]. The block diagram of existing system to diagnose Chronic Kidney Disease which is presently practiced in the clinic is shown in figure.1. the doctor considers the symptoms, case history, laboratory data and based on this he will diagnose and treat the patient.

Shortcomings of the Existing System are:

1. Time consuming

2. Uncertainty in Decision Making

3. Possibility of human errors

4. Generates incomprehensible data

\section{Proposed System}

The system that we propose here is an automatic system which computes and takes decision automatically. The block diagram of our planned system is shown in figure 2 . This model produces high quality output for a urine test. It provides a reliable performance estimate. It permits applying to unseen patients. Here, the system can collect the data from observations, laboratory testing, and the input of specific gravity of urine from MEMS and then automatically evaluates the status of SKD. In this system, since the manual prediction is eliminated, we will get more accurate results which can facilitate the right treatment and time conjointly saved.

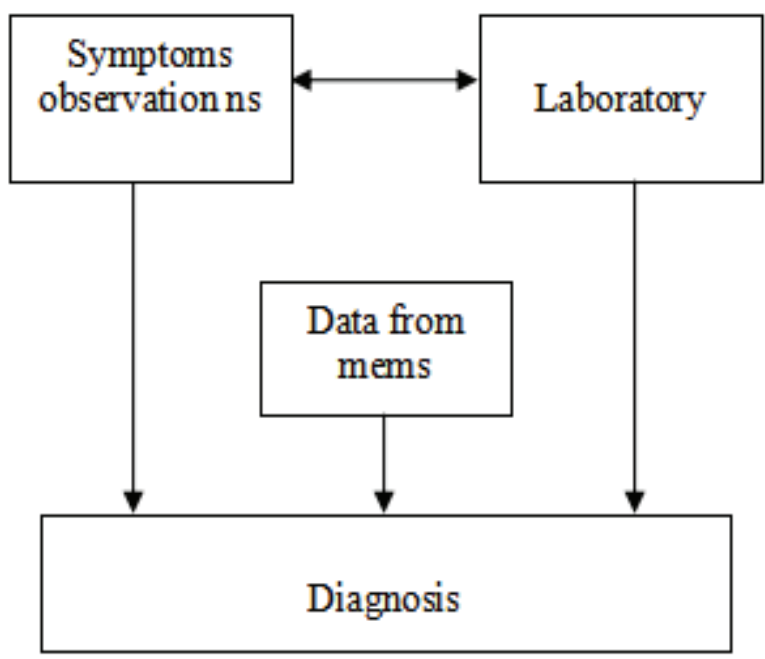

Figure 2. Proposed System

\section{MEMS}

A capacitive sensor performs well when the gap between electrodes is very small. For micro machines, a small gap leads to compressing film damping and molecular interaction throughout the motion [16]. For a resonant device, maximum damping results in low $\mathrm{Q}$ values 
(resonator quality factor) or poor signal/noise ratio. Vacuum packaging of the microtube is utilized to overcome this problem along with a compatible electrical load transfer design. This has been accomplished using vacuum wafer-towafer bonding techniques. From MEMS, measurement of specific gravity of urine is done by examining its value with typical curve obtained within the simulation of sensor[17].

\section{A. Relation of frequency of vibration of electrode of Mems and gain.}

For minimum specific gravity, the electrodes of MEMS will vibrate with maximum amplitude which is shown in figure 3. But, the amplitude of the vibration of the electrode is functionally related to specific gravity which is the underlying principle of MEMS [18]. Both these phenomena have been simulated by MATLAB 7.0. Different curves of various resonant frequencies representing different urine samples have been simulated in MATLAB. Data obtained from MATLAB are decoded so that it can be fed to software for further examination and correlation with the data of blood samples obtained from the laboratory corresponding to the status of the subject.

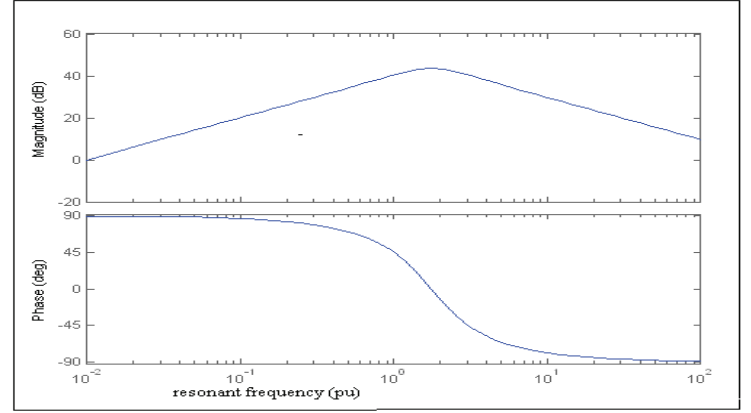

Figure 3. Relation of frequency Vs gain

B. Relation of specific gravity of liquid and output voltage of Mems

As the specific gravity of the liquid increases, the density of the liquid also increases, and thus the mass of the liquid increases [19]. With different specific gravity i.e., different concentrations of liquid, the output voltage of Mems will vary according to the curve shown in figure 4

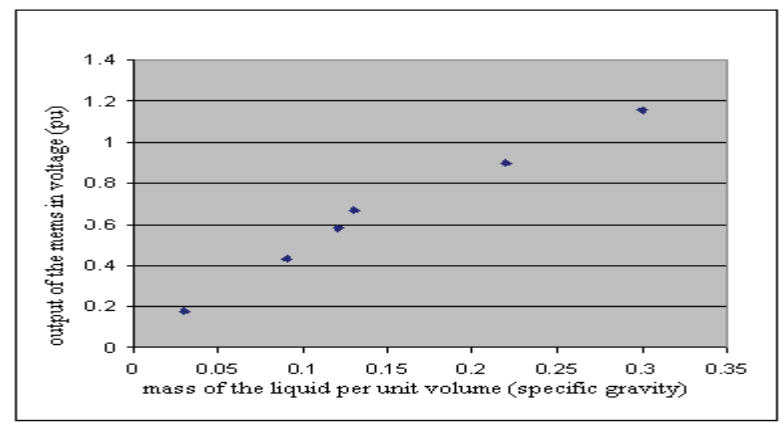

Figure 4. Relation of specific gravity of liquid Vs output of MEMS

\section{Relation of frequency and $\Delta f$}

Different curves of various resonant frequencies representing different urine samples have been simulated in MATLAB. A relation between frequency(resonant frequencies) and $\Delta \mathrm{f}$ (urine samples) has been plotted after studying different curves. The relation obtained is as shown in figure 5 .

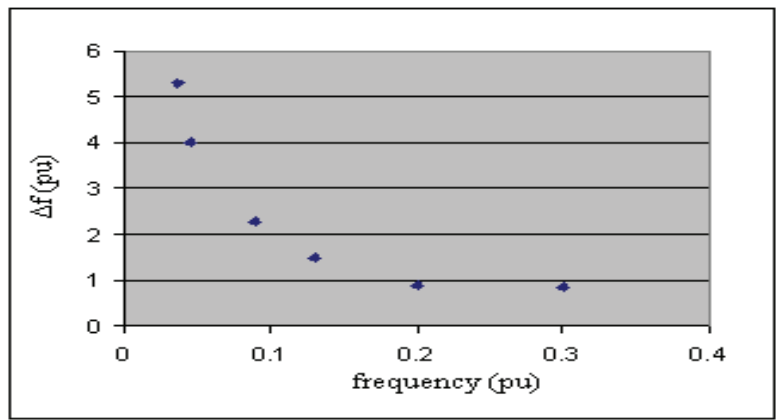

Figure 5. Relation of resonant frequency(f) Vs urine $\operatorname{samples}(\Delta \mathrm{f})$

\section{Relation of mass of the urine (Sp. gravity) and resonant frequency}

The relation of mass of the urine (Sp. Gravity) and resonant frequency is obtained using MATLAB and is shown in figure 6.

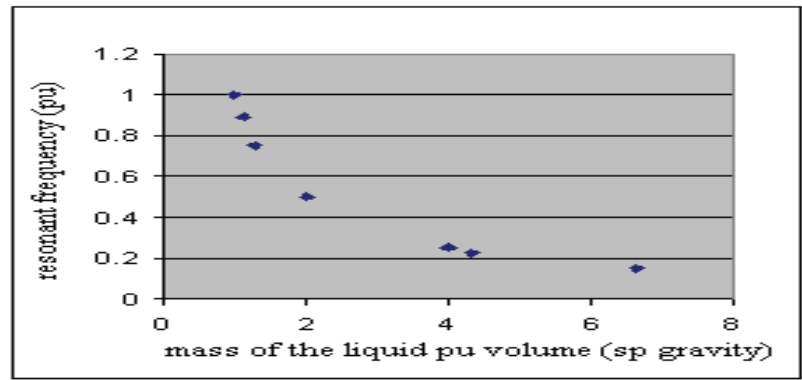

Figure 6. Relation of mass of the liquid and resonant frequency

\section{E. MATLAB simulation for renal disorder:}

The curves have been simulated in MATLAB Simulink 7.0. In this, we have simulated the curves related to the specific gravity of the urine linked to bacterial infection and this simulation is in agreement with the clinical values [20]. We have simulated the curves in a normalized way for projecting the status of the subject suffering from repeated bacterial infection. There is a possibility of renal failure with a repeated and very late bacterial infection.

A typical block diagram involved in Simulink 7.0 is shown in figure 7. 


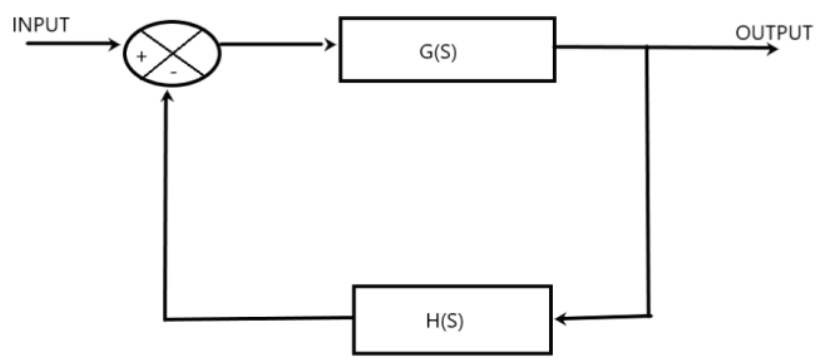

Figure 7. Block diagram in Simulink 7.0

\section{DATA Mining}

With computerization in hospitals, a large quantity of data is collected. While human decision-making is commonly best, it's poor once there are enormous amounts of data to be classified. Medical data mining has great potential for exploring hidden patterns within the data sets of the medical domain. These patterns can be used for clinical diagnosis[21]. The Parenchymal lesion is caused by a repeated bacterial infection within the urinary tract. The status of diseases related to bacterial infection has been classified in data mining through the utilization of DTREG software. Data mining is a powerful statistical analysis program that generates classification and regression decision trees that model data and can be used to predict values [22]. Data mining has been done by the DTREG software which is a robust application that is installed easily on any Windows system [23]. It provides predictive modeling and analysis software that creates decision trees, Support Vector Machine, and many more. It reads Comma Separated Value (CSV) data files that are well created from almost any data source. Here, using DTREG the classification of trees has been done through a particular option (single tree mode) present in the software where the target variable being predicted is categorical and degenerating.

\section{ConClusions}

Adults generally have a specific gravity of urine in the range of 1.010 to 1.029 . The results which are tabulated in table 1 indicate that there is a possibility of renal failure for a repeated late bacterial infection when the specific gravity is greater than 1.029. An increase in specific gravity means an increased concentration of solutes in the urine. It may cause dehydration, diarrhea, emesis, excessive sweating, urinary tract/bladder infection. When the specific gravity is less than 1.029 and approaching 1.00 then there is no possibility of renal failure. However, the status of the kidney of the patient should be of concerned in respect of this. In case, the value goes below 1.000, may lead to serious consequences.
TABLE I.

STATUS OF VARIOUS DISEASES IN DATA MINING

\begin{tabular}{|l|l|l|l|l|l|}
\hline $\begin{array}{l}\text { Data } \\
\text { size }\end{array}$ & $\begin{array}{l}\text { Sp.Gra } \\
\text { vity of } \\
\text { Urine }\end{array}$ & $\begin{array}{l}\text { Status of } \\
\text { Kidney }\end{array}$ & $\begin{array}{l}\text { Accurate } \\
\text { Classific } \\
\text { ation\% }\end{array}$ & $\begin{array}{l}\text { Mis- } \\
\text { Classif } \\
\text { ication } \\
\text { \% }\end{array}$ & $\begin{array}{l}\text { Accu } \\
\text { racy } \\
\text { \%o }\end{array}$ \\
\hline 53 & $\begin{array}{l}<=1.00 \\
9\end{array}$ & Early BI* & 96.16 & 3.84 & $\begin{array}{l}92.3 \\
2\end{array}$ \\
\hline 86 & $>1.009$ & Late BI* & 100 & 0 & 100 \\
\hline 104 & $>1.029$ & $\begin{array}{l}\text { Renal } \\
\text { failure }\end{array}$ & 91.62 & 8.38 & $\begin{array}{l}83.2 \\
4\end{array}$ \\
\hline 47 & $<1.000$ & $\begin{array}{l}\text { No Renal } \\
\text { failure }\end{array}$ & 94.12 & 5.88 & $\begin{array}{l}88.2 \\
4\end{array}$ \\
\hline
\end{tabular}

(* BI- Bacterial Infection)

The examination has been carried out in DTREG software and MATLAB. The DTREG software employs the BlackBox approach which means that the end-user is not able to see intermediate results. It uses a fixed set of in-built functions for classification. Also, there are various other constraints as a result of which DTREG gives misclassification around $5.00 \%$. But the proposed approach is flexible and gives more accurate results. This research can also be extended in the early diagnosis of renal diseases which might be of great satisfaction to the subject with appropriate modern treatment of the concerned disease. This analysis may help the urologist in decision-making in respect of the treatment of the patient.

\section{REFERENCES}

[1] Ganguli A. "Uddanam nephropathy/regional nephropathy in India: preliminary findings and a plea for further research" Am J Kidney Dis. 2016 Sep;68(3):344-8J. Clerk Maxwell, $A$ Treatise on Electricity and Magnetism, $3^{\text {rd }}$ ed., vol. 2. Oxford: Clarendon, 1892, pp.68-73.

[2] Parvati Sai Arun PV, Obula Reddy C, Akhter Y. "Uddanam kidney nephropathy under the light of metagenomics perspective" SN Compr Clin Med. 2019;1(1):23-5.

[3] Gadde P, Sanikommu S, Manumanthu R, Akkaloori “A. Uddanam nephropathy in India: a challenge for epidemiologists" Bull World Health Organ. 2017 Dec;95(12):848-9.

[4] Global Facts: About Kidney Disease - The National Kidney Foundation; https://www.kidney.org/kidneydisease/globalfacts-about-kidney-disease.

[5] Salekin A., Stankovic J. "Detection of Chronic Kidney Disease and Selecting Important Predictive Attributes". In: In Healthcare Informatics (ICHI), IEEE International Conference. 2016;262-270.

[6] SrinivasTadigadapaandSonbolMassoud-Ansari. "Applications of High-Performance MEMS Pressure Sensors Based on Dissolved Water Process". Integrated Sensing Systems, Inc.

[7] Arora M., Sharma E. A. "Chronic Kidney Disease Detection by Analyzing Medical Datasets" Weka. Int. J. Computer. Appl. 2016; 6:20-26.

[8] Di Noia T., Ostuni V. C., Pesce F et al. "An end stage kidney disease predictor based on an artificial neural network" ensemble. Expert Syst. Appl. 2013; 40:4438-4445.

[9] Gunasundari S., Janakiraman S., Meenambal S. "Velocity Bounded Boolean Particle Swarm Optimization for improved feature selection in liver and kidney disease diagnosis.” Expert Syst. Appl. 2016; 56:28-47. 
[10] Salekin A., Stankovic J. "Detection of Chronic Kidney Disease and Selecting Important Predictive Attributes", In Healthcare Informatics (ICHI), IEEE International Conference. 2016;262-270.

[11] Arora M., Sharma E. “A. Chronic Kidney Disease Detection by Analyzing Medical Datasets" in Weka. Int. J. Computer. Appl. 2016; 6:20-26.

[12] Rubini L. j., Eswaran P. "Generating comparative analysis of early-stage prediction of Chronic Kidney Disease" Int. J. Mod. Eng. Res. 2015; 5:49-55.

[13] Gadaras I., Mikhailov L. "An interpretable fuzzy rule-based classification methodology for medical diagnosis" Artif. Intell. Med. 2009; 47:25-41.

[14] Krause M., Samoylenko A., Vainio S. J. "Exosomes as renal inductive signals in health and disease, and their application as diagnostic markers and therapeutic agents", Front. cell Dev. Biol. 2015;3: 65.

[15] Jella K. K., Yu L., Yue Q et al. "Exosomal GAPDH from proximal tubule cells regulate $\mathrm{ENaC}$ activity", PLoS One. 2016; 11:1-20.

[16] Moyer, V. A. \& Force, U. S. P. S. T. "Screening for chronic kidney disease" U.S. Preventive Services Task Force recommendation statement. Ann. Intern. Med. 157, 567-570 (2012).

[17] Most Interesting Medical MEMS and Sensor Projects. MEMS Journal, Inc. Technology. Aug 7, 2014.

[18] D. Sparks*, S. Massoud-Ansari, M. Straayer, R. Smith, R. Schneider, J. Cripe, Y. Zhang, G. Meng "A Density / Specific Gravity Meter Based on Silicon Microtube Technology (MEMS)" -Proceedings Sensors Expo, Fall 2002, Boston, MA 2002

[19] NIH, "Urine specific gravity," Medical Encyclopedia, MedlinePlus, www.nim.nih.gov/medlineplus/ency, 2002.

[20] A book by Devendra Chaturvedi, "Modelling and Simulation of Systems using MATLAB and Simulink' CRC Press, Newyark, 2002

[21] Karaganda, A. G.; A.S. Manjunath; M.A Jayaram "Application of Genetic Algorith Optimized Neural Network Connection Weighs for Medical Diagnosis of Pima Indians Diabetes" International Journal on Soft Computing (IJSC), Vol. 2, No.2, May 2011

[22] Chen T, Guestrin C. XG Boost "A Scalable Tree Boosting System". In: Proceedings of the 22nd ACMSIGKDD International Conference on Knowledge Discovery and Data Mining. KDD'16. New York, NY, USA: ACM; 2016. p. 785 794.

[23] dRubini L. j., Eswaran P. "Generating comparative analysis of early-stage prediction of Chronic Kidney Disease" Int. J. Mod. Eng. Res. 2015; 5:49-55. 